\title{
EXPLICIT ENERGY-MINIMIZERS OF INCOMPRESSIBLE ELASTIC BRITTLE BARS UNDER UNIAXIAL EXTENSION
}

\author{
CARLOS MORA-CORRAL
}

\begin{abstract}
A rectangular bar made of a hyperelastic, but brittle, incompressible homogeneous and isotropic material is subject to uniaxial extension. We prove that the energy minimizers are, depending on the toughness coefficient of the material, either the homogeneous deformation, or the family of deformations for which a horizontal fracture breaks the material in two rectangular pieces, each of which is a rigid motion of the undeformed piece.
\end{abstract}

\section{INTRODUCTION}

Let $\Omega:=(-R, R) \times(0, L)$ be the reference configuration of a homogeneous, isotropic, incompressible, hyperlastic material. Sivaloganathan and Spector [8] proved that, under some natural conditions on the stored-energy function, the absolute minimizer subject to uniaxial extension is the homogeneous deformation.

In this paper, we allow the material to undergo brittle fracture. The natural space to pose the problem is the space $S B V$ of special bounded variation, and the energy functional of a deformation $u \in \operatorname{SBV}\left(\Omega, \mathbb{R}^{2}\right)$ is defined as

$$
I[u]:=\int_{\Omega} \Phi(|\nabla u(x, y)|) \mathrm{d} x \mathrm{~d} y+\kappa \mathcal{H}^{1}\left(J_{u}\right),
$$

where $\kappa>0$ is the toughness coefficient of the material, $J_{u}$ stands for the set of jump discontinuities of $u$, and $\mathcal{H}^{1}$ denotes the 1-dimensional Hausdorff measure (see [2] for properties of $S B V$ functions and applications to free discontinuity problems). Here $\nabla u$ denotes the absolutely continuous part of the distributional gradient of $u$, and the norm $|\cdot|$ of a matrix is taken as the square root of the sum of the squares of its components. The fact that the stored-energy function only depends on the norm of the deformation gradient follows from standard representation theorems, since, as well as the frameindifference requirement, our material is assumed to be isotropic and incompressible. Hence $\int_{\Omega} \Phi(|\nabla u|)$ represents the elastic energy of the deformation. The function $\Phi$ is assumed to be convex and increasing, as usual when dealing with polyconvex functions (see [3]).

We impose the boundary conditions

$$
u_{2}(x, 0)=0, \quad u_{2}(x, L)=\lambda L, \quad \text { a.e. } x \in(-R, R),
$$

where the equalitity is taken in the sense of traces, and $u=\left(u_{1}, u_{2}\right)$. Here $\lambda>1$ is a given number, and, hence, (2) models uniaxial extension of the material. We impose no boundary condition on the remaining part of the boundary. To avoid the trivial non-uniqueness of minimizers given by horizontal translations, we impose additionally

$$
\int_{\Omega} u_{1}(x, y) \mathrm{d} x \mathrm{~d} y=0 .
$$

We prove that, depending on the parameter $\kappa$, the minimizers of $I$ are either the homogeneous deformation, or the family of deformations for which a horizontal fracture breaks the material in two rectangular pieces, each of which is just a translation or a $\pi$-rotation of the undeformed piece. To be precise, the homogeneous deformation is given by

$$
u^{H}(x, y):=\left(\frac{1}{\lambda} x, \lambda y\right), \quad(x, y) \in \Omega,
$$


whereas, for each $y_{0} \in(0, L)$ and $\alpha \in \mathbb{R}$, we have the following family of horizontal-fracture deformations at level $y_{0}$ :

$$
\begin{aligned}
& u^{F_{1}, y_{0}, \alpha}(z):=\left\{\begin{array}{ll}
z+\left(\alpha\left(L-y_{0}\right), 0\right) & \text { if } z \in \Omega_{y_{0}}^{-}, \\
z+\left(-\alpha y_{0},(\lambda-1) L\right) & \text { if } z \in \Omega_{y_{0}}^{+},
\end{array} \quad u^{F_{2}, y_{0}, \alpha}(z):= \begin{cases}-z+\left(\alpha\left(L-y_{0}\right), 0\right) & \text { if } z \in \Omega_{y_{0}}^{-}, \\
z+\left(-\alpha y_{0},(\lambda-1) L\right) & \text { if } z \in \Omega_{y_{0}}^{+},\end{cases} \right. \\
& u^{F_{3}, y_{0}, \alpha}(z):=\left\{\begin{array}{ll}
z+\left(\alpha\left(L-y_{0}\right), 0\right) & \text { if } z \in \Omega_{y_{0}}^{-}, \\
-z+\left(-\alpha y_{0},(\lambda+1) L\right) & \text { if } z \in \Omega_{y_{0}}^{+},
\end{array} u^{F_{4}, y_{0}, \alpha}(z):= \begin{cases}-z+\left(\alpha\left(L-y_{0}\right), 0\right) & \text { if } z \in \Omega_{y_{0}}^{-}, \\
-z+\left(-\alpha y_{0},(\lambda+1) L\right) & \text { if } z \in \Omega_{y_{0}}^{+} .\end{cases} \right.
\end{aligned}
$$

We have denoted $\Omega_{y_{0}}^{-}:=(-R, R) \times\left(0, y_{0}\right)$ and $\Omega_{y_{0}}^{+}:=(-R, R) \times\left(y_{0}, L\right)$. All maps $u^{F_{i}, y_{0}, \alpha}$ represent realistic deformations, in the sense that all are one-to-one. However, the boundary conditions (2) model uniaxial extension only for $u^{F_{1}, y_{0}, \alpha}$.

The proof, which of course uses many ideas of Sivaloganathan and Spector [8], also depends on the rigidity result for $S B V$ deformations due to Chambolle, Giacomini and Ponsiglione [4], and on the structure theorem on the boundary of sets of finite perimeter by Federer [7].

\section{MAIN THEOREM}

The following result follows at once from polar decomposition. Recall that $S O(2)$ denotes the set of orthogonal $2 \times 2$ matrices with positive determinant.

Lemma 1. Let $A \in \mathbb{R}^{2 \times 2}$ satisfy $\operatorname{det} A=1$. Then $|A| \geq \sqrt{2}$, with equality if and only if $A \in S O(2)$.

Theorem 2. Let $\Phi:[\sqrt{2}, \infty) \rightarrow \mathbb{R}$ be a convex and strictly increasing function. Let

$$
R>0, \quad L>0, \quad \lambda>1, \quad \kappa>0, \quad \kappa_{0}:=L\left(\Phi\left(\sqrt{\lambda^{2}+\lambda^{-2}}\right)-\Phi(\sqrt{2})\right) .
$$

Let $\Omega:=(-R, R) \times(0, L)$. Let $\mathcal{A}$ be the set of $u \in S B V\left(\Omega, \mathbb{R}^{2}\right)$ such that $\operatorname{det} \nabla u=1$ a.e., and conditions

(2) and (3) hold. Define $I$ as in (1). Then the following assertions hold:

i) If $\kappa<\kappa_{0}$ then the set of minimizers of $I$ in $\mathcal{A}$ is $\left\{u^{F_{i}, y_{0}, \alpha}: i \in\{1,2,3,4\}, y_{0} \in(0, L), \alpha \in \mathbb{R}\right\}$.

ii) If $\kappa=\kappa_{0}$ then the set of minimizers of $I$ in $\mathcal{A}$ is $\left\{u^{F_{i}, y_{0}, \alpha}: i \in\{1,2,3,4\}, y_{0} \in(0, L), \alpha \in \mathbb{R}\right\} \cup$ $\left\{u^{H}\right\}$.

iii) If $\kappa>\kappa_{0}$ then the set of minimizers of $I$ in $\mathcal{A}$ is $\left\{u^{H}\right\}$.

Proof. An immediate computation shows that, for any $i \in\{1,2,3,4\}$, any $y_{0} \in(0, L)$ and any $\alpha \in \mathbb{R}$,

$$
I\left[u^{F_{i}, y_{0}, \alpha}\right]=2 R L \Phi(\sqrt{2})+2 R \kappa, \quad I\left[u^{H}\right]=2 R L \Phi\left(\sqrt{\lambda^{2}+\lambda^{-2}}\right) .
$$

Let $u \in \mathcal{A}$. We first show that $I[u]$ is bounded from below by a convex combination of $I\left[u^{F_{i}, y_{0}, \alpha}\right]$ and $I\left[u^{H}\right]$. Define $F_{u}$ as the set of $x \in(-R, R)$ such that $J_{u(x, \cdot)} \neq \varnothing$. By slicing properties of $B V$ functions (see, e.g., [2, Sect. 3.11]), $u(x, \cdot) \in S B V\left((0, L), \mathbb{R}^{2}\right)$ for a.e. $x \in(-R, R)$, and, in particular, $u(x, \cdot) \in W^{1,1}\left((0, L), \mathbb{R}^{2}\right)$ for a.e. $x \in(-R, R) \backslash F_{u}$. Moreover, by [2, Rk. 3.109],

$$
J_{u(x, \cdot)}=\left\{y \in(0, L):(x, y) \in J_{u}\right\} \quad \text { a.e. } x \in(-R, R) .
$$

Reasoning as in [8], we obtain that

$$
\int_{(-R, R) \backslash F_{u}} \int_{0}^{L} \Phi(|\nabla u(x, y)|) \mathrm{d} y \mathrm{~d} x \geq\left(2 R-\mathcal{L}^{1}\left(F_{u}\right)\right) L \Phi\left(\sqrt{\lambda^{2}+\lambda^{-2}}\right) .
$$

Here $\mathcal{L}^{1}$ denotes the Lebesgue measure over $\mathbb{R}$. The proof of [8] assumed that the deformation is of class $C^{1}$, but standard slicing techniques show that the result also holds for Sobolev maps. Let $\pi: \mathbb{R}^{2} \rightarrow \mathbb{R}^{\text {be }}$ the projection onto the first coordinate. Using that projections have Lipschitz constant 1, and equation (5), we obtain that

$$
\mathcal{H}^{1}\left(J_{u}\right) \geq \mathcal{L}^{1}\left(\pi J_{u}\right)=\mathcal{L}^{1}\left(\left\{x \in(-R, R):(\{x\} \times(0, L)) \cap J_{u} \neq \varnothing\right\}\right)=\mathcal{L}^{1}\left(F_{u}\right) .
$$


Therefore, thanks to Lemma 1,

$$
\int_{F_{u} \times(0, L)} \Phi(|\nabla u|) \mathrm{d} x \mathrm{~d} y+\kappa \mathcal{H}^{1}\left(J_{u}\right) \geq \mathcal{L}^{1}\left(F_{u}\right) L \Phi(\sqrt{2})+\kappa \mathcal{L}^{1}\left(F_{u}\right) .
$$

Putting (6), (8) and (4) together, we conclude that

$$
I[u] \geq \mathcal{L}^{1}\left(F_{u}\right)\left(\kappa-\kappa_{0}\right)+2 R L \Phi\left(\sqrt{\lambda^{2}+\lambda^{-2}}\right)=\frac{\mathcal{L}^{1}\left(F_{u}\right)}{2 R} I\left[u^{F_{i}, y_{0}, \alpha}\right]+\left(1-\frac{\mathcal{L}^{1}\left(F_{u}\right)}{2 R}\right) I\left[u^{H}\right] .
$$

for any $i \in\{1,2,3,4\}$, any $y_{0} \in(0, L)$ and any $\alpha \in \mathbb{R}$. This shows that $u^{F_{i}, y_{0}, \alpha}$ is a minimizer when $\kappa \leq \kappa_{0}$, and that $u^{H}$ is a minimizer when $\kappa \geq \kappa_{0}$. We prove now that no more minimizers can exist.

Let $\kappa<\kappa_{0}$. Then $I\left[u^{F_{i}, y_{0}, \alpha}\right]<I\left[u^{H}\right]$ for any $i \in\{1,2,3,4\}$, any $y_{0} \in(0, L)$ and any $\alpha \in \mathbb{R}$. Let $u \in \mathcal{A}$ satisfy $I[u]=I\left[u^{F_{i}, y_{0}, \alpha}\right]$. By $(9),(7),(8)$ and Lemma 1 , we obtain that $\mathcal{H}^{1}\left(J_{u}\right)=\mathcal{L}^{1}\left(F_{u}\right)=2 R$ and $\nabla u \in S O(2)$ a.e. In order to apply the area formula (see, e.g., [2, Th. 2.91]), we notice that for $\mathcal{H}^{1}$-a.e. $z \in J_{u}$, a unit vector $e(z) \in \mathbb{R}^{2}$ spans the approximate tangent space $T_{z} J_{u}$ of $J_{u}$ at $z$. Therefore, the tangential differential of $\pi$ along $J_{u}$ at $z$ is given by $\pi e(z)$. By the area formula and (5), we obtain that

$$
2 R=\mathcal{H}^{1}\left(J_{u}\right) \geq \int_{J_{u}}|\pi e(z)| \mathrm{d} \mathcal{H}^{1}(z)=\int_{-R}^{R} \mathcal{H}^{0}\left(\left\{y \in(0, L):(x, y) \in J_{u}\right\}\right) \mathrm{d} x \geq 2 R,
$$

and, hence, we can take $e(z)=(1,0)$ for $\mathcal{H}^{1}$-a.e. $z \in J_{u}$.

By the rigidity result of Chambolle, Giacomini and Ponsiglione [4], there exist $I \subset \mathbb{N}$ and a Caccioppoli partition (i.e., a partition made of sets of finite perimeter) $\left\{E_{i}\right\}_{i \in I}$ of $\Omega$ such that

$$
J_{u}=\bigcup_{i \in I} \partial^{*} E_{i} \cap \Omega, \quad \mathcal{H}^{1} \text {-a.e. }
$$

and

$$
u(z)=\sum_{i \in I}\left(R_{i} z+b_{i}\right) \chi_{E_{i}}(z), \quad \text { a.e. } z \in \Omega,
$$

for some $\left\{R_{i}\right\}_{i \in I} \subset S O(2)$ and $\left\{b_{i}\right\}_{i \in I} \subset \mathbb{R}^{2}$. Here, $\partial^{*}$ denotes the measure-theoretic boundary, which, for sets of finite perimeter, coincides $\mathcal{H}^{1}$-a.e. with Federer's boundary and with the reduced boundary (see, e.g., [2]). Moreover, thanks to the structure theorem of the boundary of planar sets of finite perimeter by Federer [7, 4.2.25] (see also [1, Cor. 1]), for each $i \in I$ there exist a $J_{i} \subset \mathbb{N}$ and a disjoint family $\left\{C_{i j}\right\}_{j \in J_{i}}$ of Jordan curves such that

$$
\partial^{*} E_{i}=\bigcup_{j \in J_{i}} C_{i j}, \quad \mathcal{H}^{1} \text {-a.e. }
$$

Consequently, $T_{z} C_{i j}$ is spanned by $(1,0)$ for $\mathcal{H}^{1}$-a.e. $z \in C_{i j} \cap \Omega$, every $i \in I$, and every $j \in J_{i}$. Fix $i \in I$ and $j \in J_{i}$. There exists a one-to-one Lipschitz arc-length parametrization $\gamma:\left[0, \mathcal{H}^{1}\left(C_{i j}\right)\right] \rightarrow C_{i j}$ of $C_{i j}$ (see, e.g., [1, Lemma 3]), and $T_{z} C_{i j}$ is spanned by $\gamma^{\prime}\left(\gamma^{-1}(z)\right)$ for $\mathcal{H}^{1}$-a.e. $z \in C_{i j}$ (see, e.g., [2, Prop. $2.88])$. Hence $\gamma^{\prime}(t)$ is proportional to $(1,0)$ for a.e. $t \in\left[0, \mathcal{H}^{1}\left(C_{i j}\right)\right]$ such that $\gamma(t) \in \Omega$. This implies that $C_{i j}$ describes a rectangle, with one edge contained in $\{-R\} \times[0, L]$, and another in $\{R\} \times[0, L]$. From the equality $\mathcal{H}^{1}\left(J_{u}\right)=2 R$ we obtain that there exists $y_{0} \in(0, L)$ such that $J_{u}=(-R, R) \times\left\{y_{0}\right\}, \mathcal{H}^{1}$ a.e. Applying the fact that open connected sets with finite perimeter only admit the trivial Caccioppoli partition formed by themselves (see [1, Prop. 2]) to $(-R, R) \times\left(0, y_{0}\right)$ and to $(-R, R) \times\left(y_{0}, L\right)$, we obtain that $\Omega$ admits only one Caccioppoli partition satisfying (11), namely,

$$
\left\{(-R, R) \times\left(0, y_{0}\right),(-R, R) \times\left(y_{0}, L\right)\right\} .
$$

We then conclude from (12) that there exist $R_{1}, R_{2} \in S O(2)$ and $b_{1}, b_{2} \in \mathbb{R}^{2}$ such that

$$
u(z)=\left(R_{1} z+b_{1}\right) \chi_{(-R, R) \times\left(0, y_{0}\right)}(z)+\left(R_{2} z+b_{2}\right) \chi_{(-R, R) \times\left(y_{0}, L\right)}(z), \quad \text { a.e. } z \in \Omega .
$$

Conditions (2) and (3) imply, after some calculations, that

$$
R_{1} \in\{-I, I\}, \quad b_{1}=\left(\alpha\left(L-y_{0}\right), 0\right), \quad\left(R_{2}, b_{2}\right) \in\left\{\left(I,\left(-\alpha y_{0},(\lambda-1) L\right)\right),\left(-I,\left(-\alpha y_{0},(\lambda+1) L\right)\right\},\right.
$$


for some $\alpha \in \mathbb{R}$.

Let $\kappa>\kappa_{0}$, and let $u \in \mathcal{A}$ satisfy $I[u]=I\left[u^{H}\right]$. Then, by $(9), \mathcal{L}^{1}\left(F_{u}\right)=0$, so by $(6), \mathcal{H}^{1}\left(J_{u}\right)=0$. Hence $u \in W^{1,1}\left(\Omega, \mathbb{R}^{2}\right)$, and we conclude, thanks to [8], that $u=u^{H}$.

Finally, let $\kappa=\kappa_{0}$, and let $u \in \mathcal{A}$ satisfy $I[u]=I\left[u^{H}\right]$. If $\mathcal{L}^{1}\left(F_{u}\right)=0$, we proceed as in the case $\kappa>\kappa_{0}$ to conclude that $u=u^{H}$. If $\mathcal{L}^{1}\left(F_{u}\right)=2 R$, we proceed as in the case $\kappa<\kappa_{0}$ to conclude that $u=u^{F_{i}, y_{0}, \alpha}$ for some $i \in\{1,2,3,4\}$, some $y_{0} \in(0, L)$ and some $\alpha \in \mathbb{R}$. Suppose, for contradiction, that $0<\mathcal{L}^{1}\left(F_{u}\right)<2 R$. From (6) we obtain that $|\nabla u|=\sqrt{\lambda^{2}+\lambda^{-2}}$ a.e. in $\left((-R, R) \backslash F_{u}\right) \times(0, L)$. As $u(x, \cdot) \in W^{1,1}\left((0, L), \mathbb{R}^{2}\right)$ for a.e. $x \in(-R, R) \backslash F_{u}$, we conclude, reasoning as in [8], that

$$
\nabla u(z)=\operatorname{diag}\left(\frac{1}{\lambda}, \lambda\right) \quad \text { a.e. } z \in\left((-R, R) \backslash F_{u}\right) \times(0, L),
$$

whereas from (8) and Lemma 1 we obtain that

$$
\nabla u(z) \in S O(2) \quad \text { a.e. } z \in F_{u} \times(0, L)
$$

and $\mathcal{H}^{1}\left(J_{u}\right)=\mathcal{L}^{1}\left(F_{u}\right)$. Call

$$
K:=S O(2) \cup \operatorname{diag}\left(\frac{1}{\lambda}, \lambda\right) .
$$

From (14) and (15) we obtain that $\nabla u \in K$ a.e. The result of Chaudhuri and Müller [5] provides, in particular, a rigidity estimate for Sobolev deformations with gradient in $K$. They proved an $L^{2}$ estimate, but a similar proof shows an $L^{p}$ estimate for $p \in(1, \infty)$ (see also [6], where an alternative proof of the $L^{p}$ rigidity estimate is given). Therefore, we can apply the $S B V$ rigidity result of [4, Th. 1.2] to obtain that there exist $I \subset \mathbb{N}$ and a Caccioppoli partition $\left\{E_{i}\right\}_{i \in I}$ of $\Omega$ such that equalities (11) and (12) hold for some $\left\{R_{i}\right\}_{i \in I} \subset K$ and $\left\{b_{i}\right\}_{i \in I} \subset \mathbb{R}^{2}$. As above, for each $i \in I$ there exist a $J_{i} \subset \mathbb{N}$ and a disjoint family $\left\{C_{i j}\right\}_{j \in J_{i}}$ of Jordan curves such that (13) holds. A similar reasoning as in (10) concludes that $T_{z} J_{u}$ is spanned by $(1,0)$ for $\mathcal{H}^{1}$-a.e. $z \in J_{u}$. As above, each $C_{i j}$ describes a rectangle, with one edge contained in $\{-R\} \times[0, L]$, and another in $\{R\} \times[0, L]$. This is a contradiction with the fact that $0<\mathcal{H}^{1}\left(J_{u}\right)<2 R$.

If $\Phi$ is not assumed to be strictly increasing, but only monotonically increasing, then we still obtain that $u^{H}$ is a minimizer of $I$ in $\mathcal{A}$ for $\kappa \geq \kappa_{0}$, and each $u^{F_{i}, y_{0}, \alpha}$ is a minimizer of $I$ in $\mathcal{A}$ for $\kappa \leq \kappa_{0}$, but some other minimizers may exist too.

Acknowledgements. Supported by Project MTM2009-07662 of the Spanish Ministry of Science and Innovation, and by Project PI2010-01 of the Basque Department of Education, Universities and Research.

\section{REFERENCES}

[1] L. Ambrosio, V. Caselles, S. Masnou, And J.-M. Morel, Connected components of sets of finite perimeter and applications to image processing, J. Eur. Math. Soc., 3 (2001), pp. 39-92.

[2] L. Ambrosio, N. Fusco, and D. Pallara, Functions of bounded variation and free discontinuity problems, Oxford University Press, New York, 2000.

[3] J. M. Ball, Convexity conditions and existence theorems in nonlinear elasticity, Arch. Rational Mech. Anal., 63 (1976/77), pp. 337-403.

[4] A. Chambolle, A. Giacomini, and M. Ponsiglione, Piecewise rigidity, J. Funct. Anal., 244 (2007), pp. 134 - 153.

[5] N. Chaudhuri and S. Müller, Rigidity estimate for two incompatible wells, Calc. Var. Partial Differential Equations, 19 (2004), pp. 379-390.

[6] C. De Lellis and L. SzéKelyhidi, JR., Simple proof of two-well rigidity, C. R. Math. Acad. Sci. Paris, 343 (2006), pp. 367-370.

[7] H. Federer, Geometric measure theory, Springer, New York, 1969.

[8] J. Sivaloganathan and S. J. Spector, On the global stability of two-dimensional, incompressible, elastic bars in uniaxial extension, Proc. R. Soc. A, 466 (2010), pp. 1167-1176.

E-mail address: mora@bcamath.org

BCAM - Basque Center for Applied Mathematics. Bizkaia Technology Park, Building 500, E-48160 Derio, Basque Country, Spain 